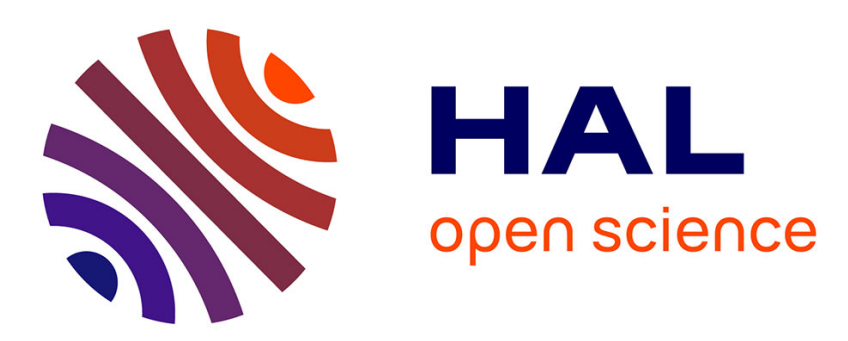

\title{
Experimental evidence of nonlinear mode coupling between spherical and nonspherical oscillations of microbubbles
}

\author{
Matthieu Guédra, Claude Inserra, Cyril Mauger, Bruno Gilles
}

\section{To cite this version:}

Matthieu Guédra, Claude Inserra, Cyril Mauger, Bruno Gilles. Experimental evidence of nonlinear mode coupling between spherical and nonspherical oscillations of microbubbles. Physical Review E , 2016, 94 (5), pp.053115. 10.1103/PhysRevE.94.053115 . hal-01516115

\section{HAL Id: hal-01516115 \\ https://hal.science/hal-01516115}

Submitted on 7 Mar 2018

HAL is a multi-disciplinary open access archive for the deposit and dissemination of scientific research documents, whether they are published or not. The documents may come from teaching and research institutions in France or abroad, or from public or private research centers.
L'archive ouverte pluridisciplinaire $\mathbf{H A L}$, est destinée au dépôt et à la diffusion de documents scientifiques de niveau recherche, publiés ou non, émanant des établissements d'enseignement et de recherche français ou étrangers, des laboratoires publics ou privés. 


\title{
Experimental evidence of nonlinear mode coupling between spherical and nonspherical oscillations of microbubbles
}

\author{
Matthieu Guédra, ${ }^{1, *}$ Claude Inserra, ${ }^{1}$ Cyril Mauger, ${ }^{2}$ and Bruno Gilles ${ }^{1}$ \\ ${ }^{1}$ Université Claude Bernard Lyon 1, INSERM, LabTAU, F-69003, Lyon, France \\ ${ }^{2}$ Université Claude Bernard Lyon 1, INSA de Lyon, CNRS, LMFA UMR 5509, F-69622 Villeurbanne CEDEX, France
}

(Received 7 July 2016; published 18 November 2016)

\begin{abstract}
We report observations of strong nonlinear interactions between the spherical, translational, and shape oscillations of micrometer-size bubbles. This is achieved through high-speed recordings of single bubble dynamics driven by amplitude-modulated ultrasound. The features of mode coupling are highlighted through (i) the exponential growth of the parametrically excited mode $(n=3)$ triggered by the spherical oscillations followed by a saturation due to energy transfer towards the translation and even modes, (ii) the excitation of modes well below their parametric pressure threshold, and (iii) clear modification of the breathing mode $R(t)$. These results are compared to recent theories accounting for nonlinear mode coupling, providing predictions in agreement with the observed bubble dynamics.
\end{abstract}

DOI: 10.1103/PhysRevE.94.053115

\section{INTRODUCTION}

When submitted to a sufficiently strong acoustic field, gas bubbles can undergo shape deformations and no longer oscillate upon a purely spherical mode. Such nonspherical surface deformations are excited by instabilities including Rayleigh-Taylor instability (acting on a very fast time scale) and Faraday (parametric) instability, which is a cumulative effect over many acoustic cycles (typically $10^{-3} \mathrm{~s}$ for $\mu \mathrm{m}$-size bubbles) [1]. For the latter, the energy transfer from the acoustically driven volume mode to the surface shape modes can be resonant either with the driving frequency or with respect to afterbounces occurring during large oscillations, with a particular interest in sonoluminescence studies [1-3]. Less appreciated than uncoupled surface instabilities induced by volume oscillations is the possibility of nonlinear coupling between spherical, translational, and surface modes [4-6]. Theoretical investigations have been devoted first to surfaceto-volume transfer in the context of sound emission by bubbles as a source of underwater sound $[7,8]$ and second to surface-to-translation transfer to explain the dancing bubble phenomenon [9]. Indirect observation of nonlinear mode coupling was performed by acoustic noise measurement of released asymmetrical bubbles [10] and evidence of multiple surface mode generation in an acoustic field has been given on millimetric bubbles [11]. Only a few studies have been done to investigate nonlinear mode coupling of micrometersize bubbles, while the enhancement of spherical oscillations induced by shape distortions is of interest in the context of medical imaging by ultrasound contrast agents [12] and microstreaming generation by tethered microbubbles [13-16]. Recently, direct observations of nonlinear mode coupling on ultrasound contrast agents have been attributed to the shell elasticity [12], while uncoated microbubbles in a strong acoustic field did not reveal such behavior [17]. In this paper, we describe a set of controlled experiments on uncoated micrometer-size bubbles in a sufficiently strong acoustic field that exhibit nonlinear mode coupling. We give evidence of

\footnotetext{
*matthieu.guedra@inserm.fr
}

shape-to-volume mode modification, and highlight the validity of the most recent theoretical developments [18-20] in the field for moderate energy transfer between modes.

\section{EXPERIMENTAL SETUP AND OBSERVATIONS}

Single air bubbles of size ranging from 30 to $80 \mu \mathrm{m}$ are nucleated by a focused laser pulse $(\lambda=532 \mathrm{~nm}$, pulse duration $5 \mathrm{~ns}$ ) in a closed 12-cm-edge cubic water tank. Distilled undergassed water is used, avoiding the rapid dissolution of the bubbles. An ultrasonic plane transducer (SinapTec, active area $\varnothing 35 \mathrm{~mm}$ ) located on the top of the tank allows one to trap the bubble in an acoustic field of frequency $f_{a}=$ $33.20 \mathrm{kHz}$. Similarly to previous works investigating shape deformations of drops [21-24] and bubbles [11,25,26], bubbles are insonified by a slowly varying amplitude-modulated signal of modulation frequency $f_{M} \ll f_{a}$, which allows one to generate the shape modes periodically. This point will be further discussed. Shadowgraphic images of the bubble are obtained using a continuous light source (LED) and high-speed recordings of the bubble dynamics are achieved using a CCD camera (Vision Research, Phantom V12.1) and a $12 \times$ objective lens (Navitar, 12x zoom), with an acquisition rate of $180 \times 10^{3}$ frames per second (180 kfps). Movies are captured with a frame size of $128 \times 128$ pixels. Typical examples of the recorded series of pictures are shown in Fig. 1 for bubbles of radii $48 \mu \mathrm{m}$ [Fig. 1(a)], $68 \mu \mathrm{m}$ [Fig. 1(b)], and $57 \mu \mathrm{m}$ [Fig. 1(c)], revealing the onset and extinction of the surface modes $n=2,3,4$, respectively. Each series of 18 pictures corresponds to instantaneous snapshots of the bubble on one half period of the low-frequency modulation. The modal amplitudes are obtained from the movies through an image processing procedure illustrated in Fig. 2. The bubble contour and weight center are extracted from the recorded pictures [Fig. 2(a)], and discrete values of the radial coordinate $r_{s}(\theta, t)$ of the bubble surface are determined [Fig. 2(b)]. A proper expansion of $r_{s}(\theta, t)$ as a Legendre polynomial series,

$$
r_{s}(\theta, t)=\sum_{n=0}^{N} a_{n}(t) P_{n}(\cos \theta),
$$


(a)

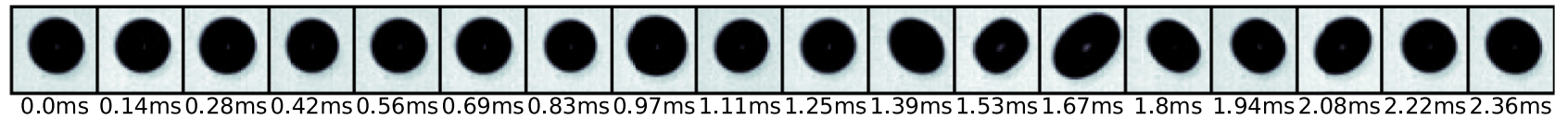

(b)

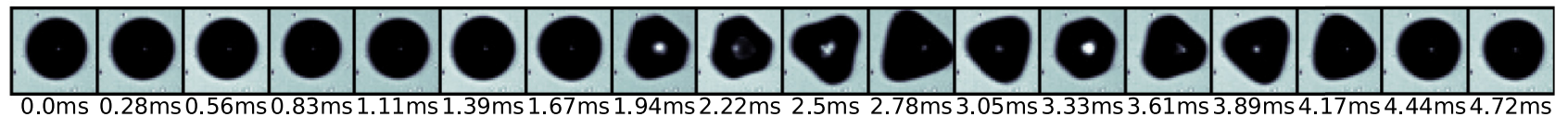

(c)

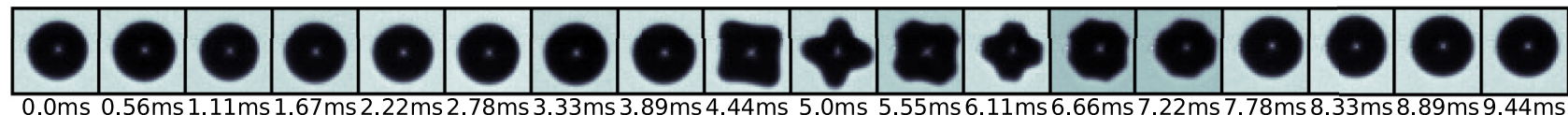

FIG. 1. Snapshot series during one half period of the low-frequency modulation, for bubbles of radii (a) 48 , (b) 68 , and (c) $57 \mu \mathrm{m}$ (see Supplemental Material [27]). The modulation frequency is different for each series: (a) $f_{M}=200$, (b) $f_{M}=100$, and (c) $f_{M}=50 \mathrm{~Hz}$.

directly leads to the computation of the modal coefficients $a_{n}(t)$ [Fig. 2(c)] by projecting $r_{s}$ on the $P_{n}$ polynomial basis, so that

$$
a_{n}(t)=\frac{2 n+1}{2} \int_{-1}^{1} r_{s} P_{n}(x) d x \quad \text { with } x=\cos \theta .
$$

Note that a reference angle $\alpha_{\text {ref }}$ might be chosen for the correct definition of $\theta$ [Fig. 2(a)]: its value fulfills an objective criterion minimizing (in a least-square sense) the difference between the rough data for $r_{s}$ and the rebuilt contour after computation of the modal coefficients [namely, the ability of the modal expansion to correctly fit the contour; see Fig. 2(b)]. We must also emphasize that $\alpha_{\text {ref }}$ is nearly constant on the whole duration of the acquisition, with typical variations of less than 1 degree (except when the bubble is in a spherical shape, where its value is obviously irrelevant).

It is worth noting that according to Eq. (1), $a_{0}$ corresponds to the breathing mode of the bubble [noted $R(t)$ in the following] and $a_{1}$ can be related to its translational motion [noted $x(t)$ in the following]. According to this modal expansion, the temporal variations of the five first modes (including $R$ and $x$ ) are given in Fig. 3 for a bubble of radius $67 \mu \mathrm{m}$. The spherical oscillations of the bubble are modulated in amplitude around
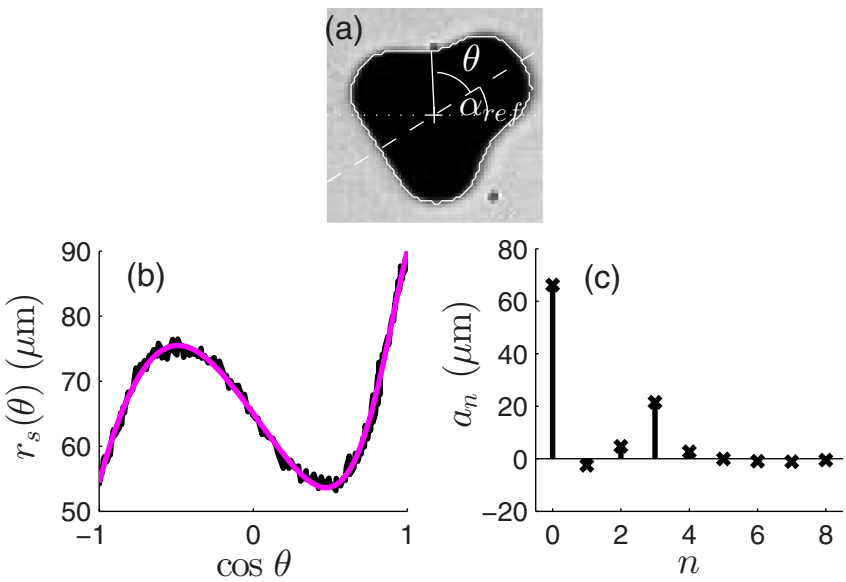

FIG. 2. Illustration of the procedure for the computation of the modal coefficients at a given time. (a) An instantaneous snapshot of the distorted bubble, together with the extracted contour and the definitions of the reference angle $\alpha_{\text {ref }}$ and the polar angle $\theta$. (b) The radial coordinate $r_{s}$ as a function of $\cos \theta$, obtained from the contour (black curve) and from the modal expansion (magenta curve) after computation of (c) the nine first coefficients $a_{n}$. the static radius, with a period $f_{M}^{-1}=10 \mathrm{~ms}$. All of the higherorder modes appear almost simultaneously when the amplitude of the breathing mode is the highest. This phenomenon is highly repeatable and the modulation of the bubble response leads to the periodic onset and extinction of the unstable modes. Particular attention is now given to one half period of the modulation in Fig. 4(a), which corresponds to a zoom-in of Fig. 3 over the first five milliseconds. During this time, the bubble, initially in a spherical shape, begins to develop some shape instabilities as its oscillations become larger, and then turns back into a spherical shape at the end of the period. The most unstable mode $(n=3)$ is parametrically excited by the breathing mode and reaches the largest amplitude of around $20 \mu \mathrm{m}$ (the bubble is mostly in a triangular shape). This mode oscillates at half the driving frequency [see Fig. 5(a)], which corresponds to the first resonance of the parametrically excited system. This is consistent with the classical linear theory

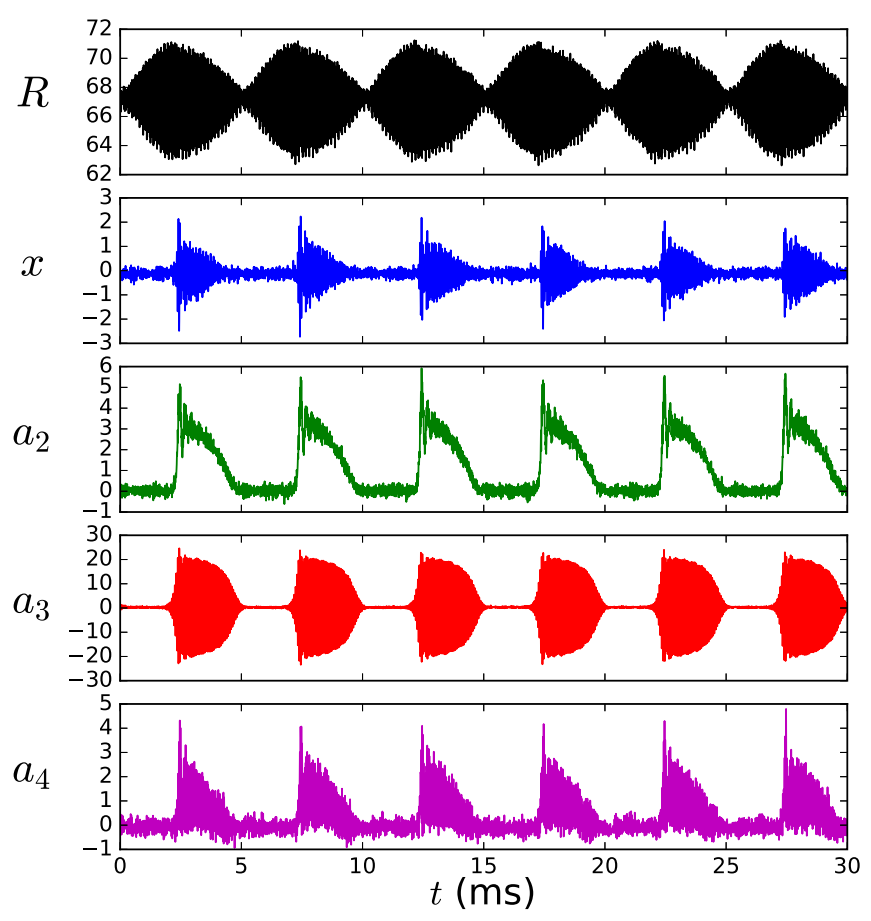

FIG. 3. Temporal evolution of the amplitudes of the radial mode $(R)$, translational mode $(x)$, and surface modes $\left(a_{i}\right)$ for a bubble of radius $67 \mu \mathrm{m}$. All quantities are given in micrometers. The modulation frequency is $f_{M}=100 \mathrm{~Hz}$. 

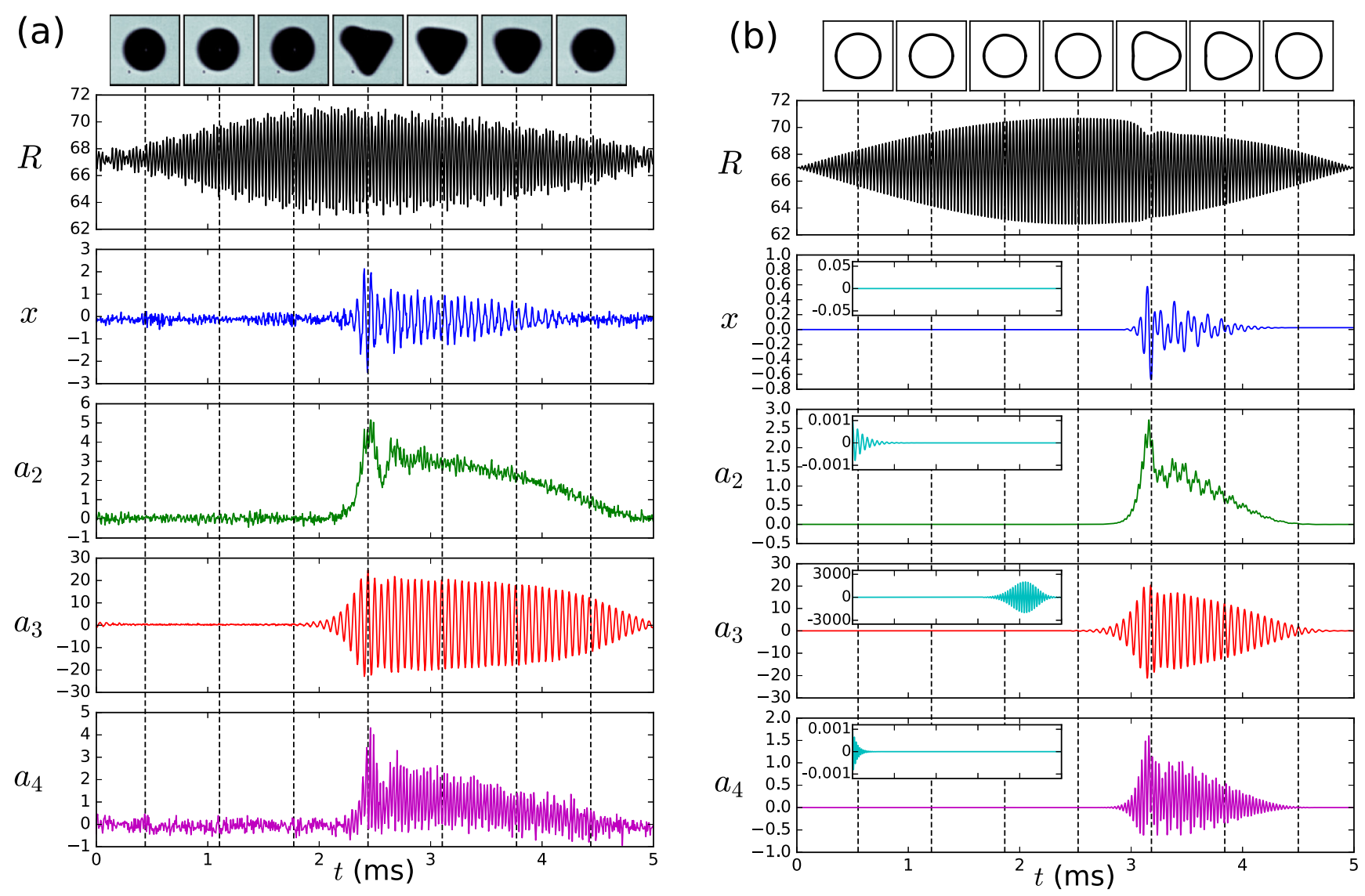

FIG. 4. Temporal evolution of the amplitudes of the radial mode $(R)$, translational mode $(x)$, and surface modes $\left(a_{i}\right)$ for a bubble of radius $67 \mu \mathrm{m}$. All quantities are given in micrometers. (a) Experimental results (zoom-in of Fig. 3). (b) Theoretical results from the coupled (main graphs) and uncoupled (inset graphs) theories. See Sec. III.

which predicts its natural frequency to be $f_{3} \simeq 15.6 \mathrm{kHz}$ for a bubble of radius $67 \mu \mathrm{m}$ [28]. Moreover, far from large
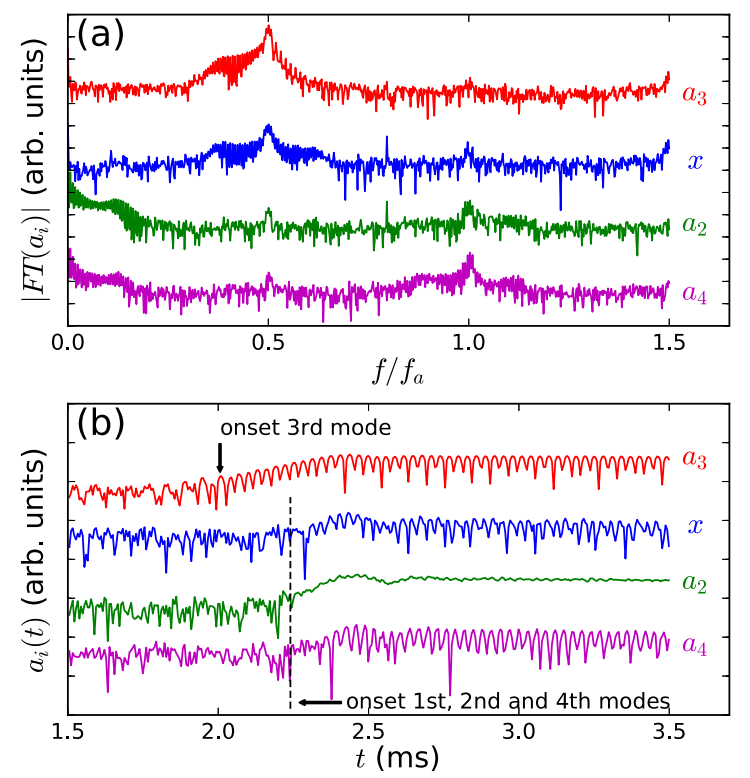

FIG. 5. (a) Fourier transform of the modal coefficients. The frequency axis is normalized by the driving frequency $f_{a}=$ $33.20 \mathrm{kHz}$. (b) Modal coefficients in logarithmic scale. Zoom-in of Fig. 4(a). The $y$-axis scales are in arbitrary units. bubble amplitudes, the linearized Rayleigh-Plesset equation allows us to infer that the driving pressure amplitude is $p_{a} \simeq 14 \mathrm{kPa}$, above the parametric threshold $p_{3 \text {,th }} \simeq 8 \mathrm{kPa}$ calculated at the driving frequency of $33.20 \mathrm{kHz}$ [29]. Once $a_{3}$ has reached its largest amplitude, it is strongly saturated and slowly decreases until its disappearance. Such a saturation is due to the energy transfer from $a_{3}$ towards the translation mode $(x)$ and the even shape modes. The first indication of this process is the time delay between the onset of $a_{3}$ and the other modes, which is easily observed when the temporal curves are plotted on a logarithmic scale [see Fig. 5(b)]. Furthermore, the even shape modes $a_{2}$ and $a_{4}$ are not driven at $f_{a} / 2$ [see Fig. 5(a)], which is far from their natural frequencies $f_{2} \simeq 8.5$ and $f_{4} \simeq 23.4 \mathrm{kHz}$. For the present bubble size and driving frequency, the corresponding pressure thresholds $p_{2, \text { th }} \simeq 38$ and $p_{4, \text { th }} \simeq 40 \mathrm{kPa}$ are much higher than the experimental acoustic pressure. Thus the oscillations of the even modes do not come from the parametric excitation of the breathing mode, but from the nonlinear coupling between nonspherical modes. A final evidence of such a nonlinear behavior is the nonzero mean value of $a_{2}$ and $a_{4}$, which is due to a nonlinear drift effect.

\section{COMPARISON WITH THEORY}

The time-resolved dynamics in Fig. 4(a) allow us to support the most recent theoretical models which consider the 
nonlinear interactions between volume oscillations, translation, and shape deformations of the bubble [18-20]. These models are based on a set of coupled second-order differential equations governing $R(t), x(t)$, and $a_{n}(t)$, obtained from a perturbative approach around the small parameter $\epsilon$ $\left(\epsilon \sim x / R \sim a_{n} / R \ll 1\right)$. The set of equations, reduced to the second order for $\epsilon$ in the present study, can be written in a condensed form as

$$
\begin{gathered}
R \ddot{R}+\frac{3}{2} \dot{R}^{2}=\frac{1}{\rho}\left(p_{\infty}+\frac{2 \sigma}{R_{0}}\right)\left(\frac{R_{0}}{R}\right)^{3 \gamma} \\
-\frac{p_{\infty}+p(t)}{\rho}-\frac{2 \sigma}{\rho R}-4 v \frac{\dot{R}}{R} \\
+\epsilon^{2} h_{0}\left(\dot{x}^{2}, a_{i}^{2}, \dot{a}_{i}^{2}, a_{i} \dot{a}_{i}, a_{i} \ddot{a}_{i}\right), \\
\epsilon\left[R \ddot{x}+3 \dot{R} \dot{x}+18 v \frac{\dot{x}}{R}\right]=\epsilon^{2} h_{1}\left(a_{i} a_{j}, a_{i} \dot{a}_{j}, \dot{a}_{i} \dot{a}_{j}\right), \\
\epsilon\left[\ddot{a}_{n}+B_{n} \dot{a}_{n}-A_{n} a_{n}\right]=\epsilon^{2} h_{n}\left(a_{i}^{2}, \dot{a}_{i}^{2}, a_{i} \dot{a}_{i}, a_{i} a_{j}, a_{i} \dot{a}_{j}\right),
\end{gathered}
$$

where $\rho$ and $v$ are the liquid density and kinematic viscosity, $\sigma$ is the surface tension, $\gamma$ is the gas polytropic index, $R_{0}$ is the bubble static radius, $p_{\infty}$ and $p(t)$ are the static part and the acoustic part of the liquid pressure, and the functions $A_{n}$ and $B_{n}$ are those involved in the uncoupled parametric equations problem and are given by [1]

$$
\begin{aligned}
& A_{n}(t)=(n-1)\left[\frac{\ddot{R}}{R}-\frac{(n+1)(n+2) \sigma}{\rho R^{3}}-(n+2)\right. \\
&\left.\times\left(1+\frac{2 n \delta}{R}\right) \frac{2 v \dot{R}}{R^{3}}\right], \\
& B_{n}(t)=3 \frac{\dot{R}}{R}+(n+2)\left[(2 n+1)-(n+2) \frac{2 n \delta}{R}\right] \frac{2 v}{R^{2}},
\end{aligned}
$$

where $\delta$ denotes the viscous boundary layer thickness. In Eqs. (3)-(5), the functions $h_{i}$ take into account the nonlinear interactions between the different modes; their mathematical expressions are not derived here but they can be found in Ref. [19]. Equations (3)-(5) also account for viscous dissipation, which is significant for micrometer-size bubbles and is expected to increase for higher-order modes $[17,30]$. Assuming that the viscous layer thickness $\delta$ is much smaller than the bubble radius, the boundary layer approximation allows one to consider the viscous damping terms in Eqs. (3)-(7) [proportional to $\left.(\delta / R)^{2} \ll 1\right]$ as perturbations, just as the nonlinear coupling terms are. Therefore, viscous dissipation is only introduced at the lowest order in $\epsilon$, while the dependence of the functions $h_{i}$ on viscosity can be discarded. Note that $\delta \simeq 2.2 \mu \mathrm{m}$ at the ultrasonic frequency used in our experiments, thus validating the boundary layer approximation for bubbles of a few tens of micrometers in radius.

Equations (3)-(5) are solved with standard numerical integration methods using a PYTHON programming language code. Figure 4(b) gives the results obtained for an acoustic driving pressure of the form

$$
p(t)=p_{a} \sin \left(2 \pi f_{M} t\right) \sin \left(2 \pi f_{a} t\right),
$$

with $p_{a}=14 \mathrm{kPa}, f_{M}=100 \mathrm{~Hz}$, and $f_{a}=33.20 \mathrm{kHz}$ corresponding to the experimental conditions of Fig. 4(a), and initial conditions $R=67 \mu \mathrm{m}, a_{2}=a_{3}=a_{4}=1 \mathrm{~nm}\left(\sim 10^{-4} R_{0}\right)$, and $\dot{R}=\dot{x}=\dot{a}_{2}=\dot{a}_{3}=\dot{a}_{4}=0$. A remarkable agreement is found both on temporal dynamics and order of magnitude [particularly for $R(t)$ and $a_{3}(t)$ ]. The model well captures the amplification of the most unstable mode $\left(a_{3}\right)$ and its saturation due to nonlinear coupling, with the appearance of the translation mode $(x)$ and the even shape modes $\left(a_{2}\right.$ and $\left.a_{4}\right)$. These modes cannot be predicted by the theory when the nonlinear interactions between modes are not taken into account, i.e., if we discard the $h_{i}$ functions in Eqs. (3)-(5) [see the inset graphs in Fig. 4(b)]. In this case, the even shape modes simply decay as they are driven well below their unstable growth threshold, while the $a_{3}$ mode is exponentially amplified until arbitrarily large values since no physical mechanisms can lead to its saturation.

\section{BREATHING MODE MODIFICATION}

A slight modification of the breathing mode $(R)$ is predicted by the theory when the higher-order modes reach their largest amplitudes, as observed in Fig. 4(b). In this case, the nonlinear terms involved in the function $h_{0}$ in Eq. (3) (especially $a_{3}^{2}$ and $\dot{a}_{3}^{2}$ here) become sufficiently large to act on the temporal dynamics of $R(t)$. This effect can hardly be observed in the experimental results shown in Fig. 4(a), and any conclusion regarding the modification of the breathing mode by shape

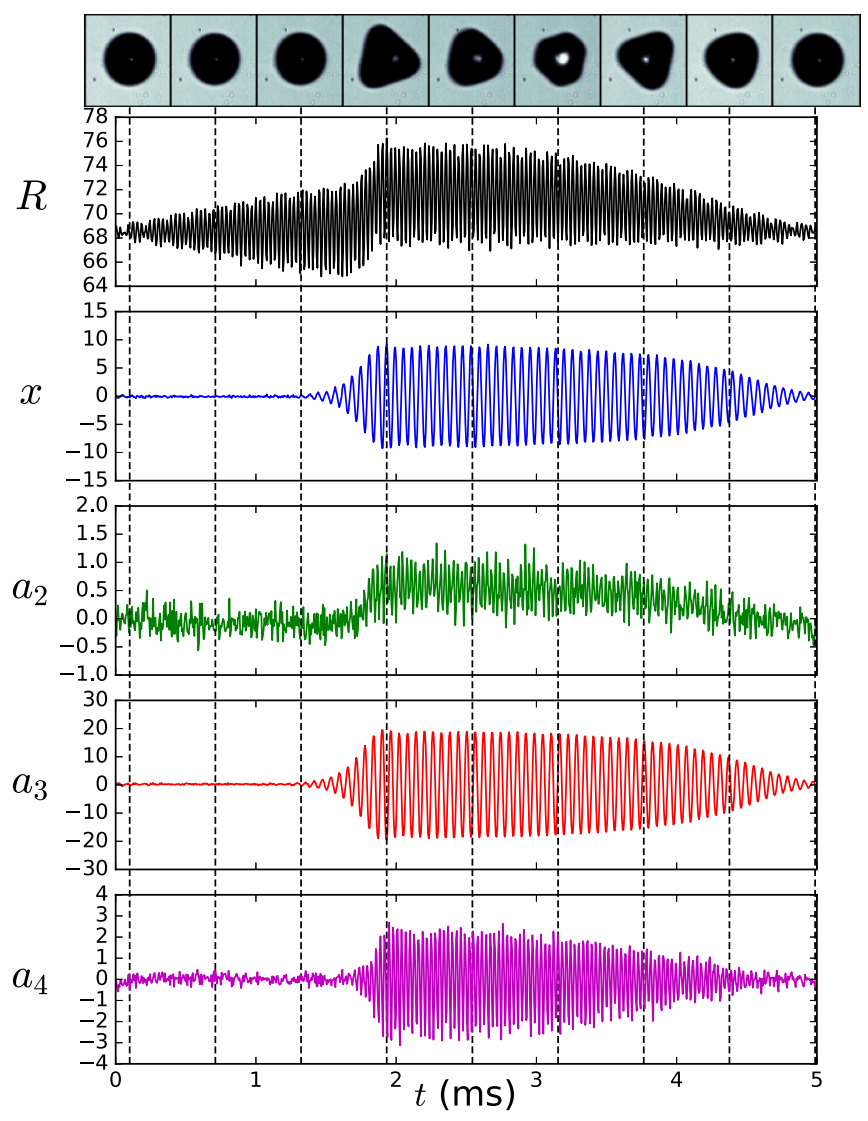

FIG. 6. Temporal evolution of the amplitudes of the radial mode $(R)$, translational mode $(x)$, and surface modes $\left(a_{i}\right)$ for a bubble of radius $68.5 \mu \mathrm{m}$. All quantities are given in micrometers. 
modes would be purely speculative at this point. However, a significant modification of $R(t)$ is demonstrated for a similar size bubble $\left(R_{0}=68.5 \mu \mathrm{m}\right)$ driven at larger amplitude $\left(p_{a} \simeq\right.$ $15.3 \mathrm{kPa}$; see Fig. 6). For this case, both the third shape mode $a_{3}$ and the translation mode $x$ exponentially grow until large amplitudes and are heavily saturated. Note that a comparison with numerical simulations is not provided for the results of Fig. 6 because too large amplitudes reached by $x(t)$ and $a_{3}(t)$ render the calculations unstable. In that case, the function $h_{0}$ in Eq. (3) is no longer a perturbation of the Rayleigh-Plesset equation and the asymptotic expansion fails.

\section{CONCLUSIONS}

We reported time-resolved dynamics of nonspherical microbubbles obtained from high-speed recordings. Suitable image processing and modal expansion of the bubble contour allowed us to determine the temporal evolution of the modes, including the breathing mode $(n=0)$, the translational mode $(n=1)$, and the shape modes $(n \geqslant 2)$. Analyzing the experimental results revealed that the excitation of several nonspherical modes does not come from the classical parametric resonance phenomenon, but from nonlinear coupling. Successful comparisons with numerical simulations confirmed our assertions, providing at the same time an experimental validation of recent theoretical developments [18-20] in the case of micrometer-size bubbles. Finally, we concluded our analysis by focusing on the shape-to-volume mode modification effect, which is of particular interest for the enhancement of ultrasound contrast agents' echogenicity [12] as well as for the manipulation of micrometric objects by bubble-induced streaming [16]. We reported such an effect for a $68.5-\mu \mathrm{m}$-size bubble undergoing both significant translational oscillations and triangular shape deformations. Our experimental results thus confirm the ability of higher-order modes to significantly alter the spherical mode of micrometric bubbles, although decisive conclusions supporting the aforementioned applications would require studies on even smaller bubbles and measurements of the scattered acoustic field and streaming flow induced by the bubble. The results presented in Fig. 6 also illustrate the limitations of the most recent models [18-20] which, because they are based on an asymptotic approach and on the assumption of small perturbations around the spherical mode, are currently unable to reproduce the complicated dynamics obtained here when several shape modes simultaneously reach amplitudes of oscillations at an order of magnitude equivalent to the spherical mode itself.

\section{ACKNOWLEDGMENTS}

This work was supported by the French National Research Agency ANR-MOST project CARIBBBOU (Grant No. ANR15-CE19-0003). The authors thank Dr. Stephen J. Shaw for useful discussions and advice.
[1] M. P. Brenner, D. Lohse, and T. F. Dupont, Phys. Rev. Lett. 75, 954 (1995).

[2] R. G. Holt and D. F. Gaitan, Phys. Rev. Lett. 77, 3791 (1996).

[3] M. P. Brenner, S. Hilgenfeldt, and D. Lohse, Rev. Mod. Phys. 74, 425 (2002).

[4] J. E. Ffowcs-Williams and Y. P. Guo, J. Fluid Mech. 224, 507 (1991).

[5] C. C. Mei and X. Zhou, J. Fluid Mech. 229, 29 (1991).

[6] Z. C. Feng and L. G. Leal, J. Fluid Mech. 226, 209 (1994).

[7] M. S. Longuet-Higgins, J. Fluid Mech. 201, 525 (1989).

[8] M. S. Longuet-Higgins, J. Fluid Mech. 201, 543 (1989).

[9] T. B. Benjamin and A. T. Ellis, J. Fluid Mech. 212, 65 (1990).

[10] Y. Mao, L. A. Crum, and R. A. Roy, J. Acoust. Soc. Am. 98, 2764 (1995).

[11] E. H. Trinh, D. B. Thiessen, and R. G. Holt, J. Fluid Mech. 364, 253 (1998).

[12] B. Dollet et al., Ultrasound Med. Biol. 34, 1465 (2008).

[13] P. Marmottant and S. Hilgenfeldt, Nature (London) 423, 153 (2003).

[14] X. Zhao, P. A. Quinto-Su, and C.-D. Ohl, Phys. Rev. Lett. 102, 024501 (2009).

[15] Y. Chen et al., Lab Chip 16, 3024 (2016).

[16] D. Ahmed et al., Nat. Commun. 7, 11085 (2016).

[17] M. Versluis, D. E. Goertz, P. Palanchon, I. L. Heitman, S. M. van der Meer, B. Dollet, N. de Jong, and D. Lohse, Phys. Rev. E 82, 026321 (2010).
[18] A. A. Doinikov, J. Fluid Mech. 501, 1 (2004).

[19] S. J. Shaw, Phys. Fluids 18, 072104 (2006).

[20] S. J. Shaw, Phys. Fluids 21, 022104 (2009).

[21] P. L. Marston and R. E. Apfel, J. Colloid Interface Sci. 68, 280 (1979).

[22] P. L. Marston and R. E. Apfel, J. Acoust. Soc. Am. 67, 27 (1980).

[23] E. Trinh, A. Zwern, and T. G. Wang, J. Fluid Mech. 115, 453 (1982).

[24] C. L. Shen, W. J. Xie, and B. Wei, Phys. Rev. E 81, 046305 (2010).

[25] T. J. Asaki and P. L. Marston, J. Fluid Mech. 300, 149 (1995).

[26] K. Ohsaka, Phys. Fluids 15, 1189 (2003).

[27] See Supplemental Material at http://link.aps.org/supplemental/ 10.1103/PhysRevE.94.053115 for high-speed recordings of oscillating bubbles mainly developing the surface modes (a) $n=2$, (b) $n=3$, and (c) $n=4$. The bubble is driven by a $33.20 \mathrm{kHz}$ amplitude-modulated ultrasonic field at a modulation frequency (a) $f_{M}=200$, (b) $f_{M}=100$, and (c) $f_{M}=50 \mathrm{~Hz}$. The movies are captured with a frame rate of $180 \mathrm{kpps}$ and displayed on one half period of the modulation.

[28] H. Lamb, Hydrodynamics, 4th ed. (Cambridge University Press, Cambridge, 1916), Chap. 9, p. 469.

[29] P. R. Birkin et al., J. Acoust. Soc. Am 130, 3297 (2011).

[30] S. Hilgenfeldt, D. Lohse, and M. P. Brenner, Phys. Fluids 8, 2808 (1996). 